\title{
Mechanisms of Cluster Formation in Force-Free Granular Gases
}

\author{
C. Salueña ${ }^{1}$, L. Almazán ${ }^{1,2}$ and N. V. Brilliantov ${ }^{3 *}$ \\ ${ }^{1}$ Department of Mechanical Engineering, Universitat Rovira i Virgili, E-43007 Tarragona, Spain \\ ${ }^{2}$ Centre de Recerca Matemàtica, 08193 Bellaterra, Spain \\ ${ }^{3}$ Department of Mathematics University of Leicester, Leicester LE1 7RH, UK
}

\begin{abstract}
The evolution of a force-free granular gas with a constant restitution coefficient is studied by means of granular hydrodynamics. We numerically solve the hydrodynamic equations and analyze the mechanisms of cluster formation. According to our findings, the presently accepted mode-enslaving mechanism may not be responsible for the latter phenomenon. On the contrary, we observe that the cluster formation is mainly driven by shock-waves, which spontaneously originate and develop in the system. This agrees with a previously suggested mechanism of formation of density singularities in one-dimensional granular gases.
\end{abstract}

Key words: granular gas, cluster instability, hydrodynamics, numerical simulations AMS subject classification: $35 \mathrm{Q} 35$

\section{Introduction}

Granular gases, being rarefied systems of dissipatively colliding particles, behave in many respect as ordinary molecular gases. Similarly to molecular gases, the intensity of random motion of grains is characterized by temperature and the velocity distribution, which is not so far from the Maxwellian distribution and the large-scale behavior of a granular gas may be well described by hydrodynamic equations [28, 26, 5]. Still however an important difference between these systems exists: Being isolated, the molecular gases keep their energy constant and stay uniform; contrarily, the granular gases not only permanently cool down due to dissipative collisions, but spontaneously

*Corresponding author. E-mail: nb144@leicester.ac.uk 
develop complicated self-organized spatio-temporal structures such as clusters [15] and vortices [10].

The dissipative collisions between particles $i$ and $j$ are characterized by the (normal) coefficient of restitution, which relates the normal component of the relative velocity before a collision, $g$, to that after the collision, $g^{\prime}$ :

$$
\varepsilon \equiv \frac{g^{\prime}}{g}=-\frac{\vec{v}_{i j}^{\prime} \cdot \vec{e}_{i j}}{\vec{v}_{i j} \cdot \vec{e}_{i j}}, \quad \quad \vec{e}_{i j} \equiv \frac{\vec{r}_{i}-\vec{r}_{j}}{\left|\vec{r}_{i}-\vec{r}_{j}\right|}
$$

with $\vec{v}_{i j} \equiv \vec{v}_{i}-\vec{v}_{j}$ and $\vec{v}_{i j}^{\prime}$ being correspondingly the pre-collisional and after-collisional relative velocities and with vector $\vec{r}_{i j} \equiv \vec{r}_{i}-\vec{r}_{j}$ joining the particle centers at the moment of the collision. Although it is known that the coefficient of restitution depends on the impact velocity, $\varepsilon=\varepsilon(g)$, $[20,9,32,24,30]$ and that the cluster and vortex formation are transient processes, due to such dependence $[6,8,27]$, we assume for simplicity that $\varepsilon$ is constant. In the present paper we focus on the mechanism of cluster formation, leaving the analysis of the cluster dissolution to future studies.

To explain the phenomenon of cluster formation, the linear stability analysis of hydrodynamic equations has been performed and it has been shown that a homogeneous force-free granular gas is unstable with respect to density fluctuations $[35,3,10,4,2,26,5]$. Qualitatively this may be understood as follows. Let a small density fluctuation arise in an initially uniform gas. This causes more frequent collisions of particles in the region, which becomes even denser as a result. Indeed, more frequent collisions cause a faster cooling of the gas, so that the gas pressure drops down in this part of the system. Then a flux of particles from nearby regions to the region of smaller pressure enhances the initial density fluctuation [15]. In this way a positive feedback for the enhancement of density homogeneities is formed. Mathematically, this mechanism is formulated in terms of the mode enslaving theory [15] (see the discussion below), which predicts the decrease of pressure with increasing density. The above explanation of cluster formation is simple, clear and hence attractive. Still, however, the direct confirmation of the mode-enslaving mechanism is lacking. The most natural way to check its validity is to study the evolution of a force-free granular gas using granular hydrodynamics, that has been successfully applied to describe many pattern formation phenomena, e.g. $[17,8,5,12,11]$. Moreover, within the hydrodynamic approach one can trace in detail the evolution of various hydrodynamic fields of interest, which is not always possible in the Molecular Dynamics simulations [25].

The aim of the present study is to explore the cluster formation in the force-free granular gases and to analyze the possible mechanisms of this phenomenon. We perform the analysis using the numerical solution of hydrodynamic equations. On contrast to previous studies, where the kinetic coefficients derived for the dilute limit were used, we apply recently obtained coefficients [22], which may be employed for large densities. The latter is important, since at the onset of the cluster formation, some parts of the gas are no more dilute and the density of clusters does not differ much from the density of hard spheres solids. We also employ a more accurate equation of state for the granular gas pressure. This may be applicable even beyond the freezing point, when the system approaches the random close packing. The paper is organized as follows. In the next Sec. II we sketch the hydrodynamic theory of granular gases, in Sec. III we discuss the mechanism of cluster formation and in Sec. IV the detail of the numerical solution of the hydrodynamic equations are 
given; here we also discuss the simulation results. Finally, in Sec. V we summarize our findings.

\section{Hydrodynamic theory of granular gases}

The hydrodynamic theory of granular gases has been developed for granular gases with constant coefficient of restitution e.g. [21, 18, 16, 33, 3, 4, 14, 2, 26, 5] and for the granular gases of viscoelastic particles $[6,7,5]$. Using the hydrodynamic theory, the cluster formation has been explored [17, 8].

For two-dimensional granular gases the hydrodynamic equations read $[2,7,5]$

$$
\begin{aligned}
\frac{\partial n}{\partial t}+\vec{\nabla} \cdot(n \vec{u}) & =0 \\
\frac{\partial \vec{u}}{\partial t}+\vec{u} \cdot \vec{\nabla} \vec{u}+(n m)^{-1} \vec{\nabla} \cdot \hat{P} & =0 \\
\frac{\partial T}{\partial t}+\vec{u} \cdot \vec{\nabla} T+\frac{1}{n}\left(P_{i j} \nabla_{j} u_{i}+\vec{\nabla} \cdot \vec{q}\right)+\zeta T & =0 .
\end{aligned}
$$

Here $n(\vec{r}), \vec{u}(\vec{r})$ and $T(\vec{r})$ denote respectively the gas number density, the flux velocity and temperature; $m$ is the mass of a grain, so that $m n(\vec{r})$ stands for the mass density. The pressure tensor $P_{i j}$ and the heat flux $\vec{q}$ read:

$$
\begin{aligned}
P_{i j} & =p \delta_{i j}-\eta\left(\nabla_{i} u_{j}+\nabla_{j} u_{i}-\delta_{i j} \vec{\nabla} \cdot \vec{u}\right)-\gamma \delta_{i j} \nabla \cdot \vec{u} \\
\vec{q} & =-\kappa \vec{\nabla} T-\mu \vec{\nabla} n
\end{aligned}
$$

where $\eta$ is the shear viscosity, $\gamma$ the bulk viscosity, $\kappa$ the thermal conductivity, and the coefficient $\mu$ does not have an analogue for a gas of elastic particles. In Eq. (2.2) $p$ is the hydrostatic pressure, which may be expressed in terms of the packing fraction of the gas $\phi=\frac{1}{4} \pi \sigma^{2} n$ ( $\sigma$ is the diameter of granular particles) as

$$
p=n T\left[1+(1+\varepsilon) \phi g_{2}(\sigma)\right],
$$

where $g_{2}(\sigma)$ is the contact value of the pair correlation function. Several formulas can be used here for $g_{2}(\sigma)$, but we have chosen the convenient formulas obtained by Torquato [36],

$$
g_{2}(\sigma)= \begin{cases}\frac{1-(7 / 16) \phi}{(1-\phi)^{2}} & \text { for } 0 \leq \phi<\phi_{f} \\ \frac{1-(7 / 16) \phi_{f}}{\left(1-\phi_{f}\right)^{2}} \frac{\phi_{c}-\phi_{f}}{\phi_{c}-\phi} & \text { for } \phi_{f} \leq \nu<\phi_{c}\end{cases}
$$

which go past the freezing point $\phi_{f}=0.69$ and approach the random close packing, $\phi_{c}=0.82$ (in two dimensions), with more accuracy than the Carnahan-Starling expression for hard discs.

The kinetic coefficients $\eta, \kappa, \mu$ and the cooling coefficient $\zeta$ sensitively depend on the particular collision model. For a dense granular gas of hard disks with constant normal restitution coefficient 
these read as follows. The cooling coefficient acquires for large densities an additional contribution proportional to the velocity field divergency $\nabla \cdot \vec{u}$ :

$$
\zeta(\varepsilon, \phi)=\zeta_{0}+\zeta_{1} \nabla \cdot \vec{u} .
$$

Here the coefficients $\zeta_{0}$ and $\zeta_{1}$ are given by

$$
\begin{aligned}
\zeta_{0} & =2 n \sigma \sqrt{\frac{\pi T}{m}} \zeta_{0}^{*}, \\
\zeta_{1} & =\frac{3}{2} \phi g_{2}(\sigma)\left(1-\varepsilon^{2}\right)\left[\frac{3}{32} \frac{\frac{1}{8} \omega^{*}-2(1+\varepsilon)\left(\frac{1}{3}-\varepsilon\right) a_{2}}{\nu_{\gamma}^{*}-\frac{3}{4}\left(1-\varepsilon^{2}\right)}-1\right]
\end{aligned}
$$

with the dimensionless coefficients, which depend on both the inelasticity and the density of the granular gas,

$$
\zeta_{0}^{*}=\frac{1}{2} g_{2}(\sigma)\left(1-\varepsilon^{2}\right)\left(1+\frac{3 a_{2}}{16}\right)
$$

and

$$
\begin{aligned}
\nu_{\gamma}^{*} & =-\frac{1+\varepsilon}{192}\left(30 \varepsilon^{3}-30 \varepsilon^{2}+153 \varepsilon-185\right), \\
\omega^{*} & =(1+\varepsilon)\left[\left(1-\varepsilon^{2}\right)(5 \varepsilon-1)-\frac{a_{2}}{6}\left(15 \varepsilon^{3}-3 \varepsilon^{2}+69 \varepsilon-41\right)\right] .
\end{aligned}
$$

The coefficient $a_{2}$ stands for the second Sonine coefficient which characterizes the shape of the velocity distribution function [5],

$$
a_{2}=\frac{16(1-\varepsilon)\left(1-2 \varepsilon^{2}\right)}{57-25 \varepsilon+30 \varepsilon^{2}(1-\varepsilon)} .
$$

The shear and bulk viscosities, $\eta$ and $\gamma$ respectively, read

$$
\begin{aligned}
& \eta(\varepsilon, \phi)=\eta_{0}\left\{\frac{\left[1-\frac{1}{4}(1+\varepsilon)(1-3 \varepsilon) \phi g_{2}(\sigma)\right]\left[1+\frac{1}{2} \phi g_{2}(\sigma)(1+\varepsilon)\right]}{\nu_{\eta}^{0}-\frac{1}{2} \zeta_{0}^{*}}+\frac{\gamma^{*}}{2}\right\}, \\
& \gamma(\varepsilon, \phi)=\eta_{0} \gamma^{*}
\end{aligned}
$$

where

$$
\gamma^{*}=\frac{8}{\pi} \phi^{2} g_{2}(\sigma)(1+\varepsilon)\left(1-\frac{a_{2}}{16}\right)
$$

and $\eta_{0}$ is the viscosity for the gas of elastic particles in the dilute limit, $\eta_{0}=1 /(2 \sigma) \sqrt{m T / \pi}$. The dimensionless coefficient $\nu_{\eta}^{*}(\varepsilon, \phi)$ is defined as

$$
\nu_{\eta}^{*}=\frac{1}{8} g_{2}(\sigma)(7-3 \varepsilon)(1+\varepsilon)\left(1-\frac{7 a_{2}}{16}\right) .
$$


The thermal conductivity is given by the expression

$$
\kappa(\varepsilon, \phi)=\kappa_{0}\left\{\kappa_{k}^{*}\left[1+\frac{3}{4} \phi g_{2}(\sigma)(1+\varepsilon)\right]+\frac{2}{\pi} \phi^{2} g_{2}(\sigma)(1+\varepsilon)\left(1-\frac{7 a_{2}}{16}\right)\right\},
$$

where $\kappa_{0}=2 / \sigma \sqrt{T /(m \pi)}$ is the ideal gas contribution and the coefficient $\kappa_{k}^{*}$ reads

$$
\kappa_{k}^{*}=\frac{1+2 a_{2}+\frac{3}{8} \phi g_{2}(\sigma)(1+\varepsilon)^{2}\left[2 \varepsilon-1+a_{2}(1+\varepsilon)\right]}{2\left(\nu_{\kappa}^{*}-2 \zeta_{0}^{*}\right)},
$$

while the coefficient $\nu_{\kappa}^{*}$ stands for

$$
\nu_{\kappa}^{*}=\frac{1}{4} g_{2}(\sigma)(1+\varepsilon)\left[1+\frac{15}{4}(1-\varepsilon)+\frac{365-273 \varepsilon}{64} a_{2}\right] .
$$

Finally, the coefficient $\mu$, coupling the density gradient and the heat flux, can be expressed as

$$
\mu=\frac{4 T}{n \sigma} \sqrt{\frac{T}{m \pi}}\left[1+\frac{3}{4} \phi g_{2}(\sigma)(1+\varepsilon)\right] \mu_{k}^{*}
$$

where

$$
\mu_{k}^{*}=\frac{\left[(1+\Delta) \zeta_{0}^{*} \kappa_{k}^{*}+a_{2} / 2+3 / 8 \phi g_{2}(\sigma)(1+\varepsilon)(1+\Delta / 2)\right]\left[\varepsilon(\varepsilon-1)+\left(a_{2} / 6\right)\left(14-3 \varepsilon+3 \varepsilon^{2}\right)\right]}{2 \nu_{\kappa}^{*}-3 \zeta_{0}^{*}}
$$

and $\Delta$ depends on the model adopted for the pair correlation function at contact. It is given in our case by

$$
\Delta=\phi \frac{\partial}{\partial \phi} \ln g_{2}(\sigma)=\left\{\begin{array}{ll}
\frac{\phi(25-7 \phi)}{(16-7 \phi)(1-\phi)} & \text { for } 0 \leq \phi<\phi_{f} \\
\frac{\phi}{\left(\phi_{c}-\phi\right)} & \text { for } \phi_{f} \leq \phi<\phi_{c}
\end{array} .\right.
$$

These coefficients have been first obtained by Lutsko [22] for a $d$ dimensional gas and have been re-derived later by Garzó [13].

Strictly speaking, the above kinetic coefficients are derived for moderate densities and it is not completely justified to use them at late stages, when dense clusters are formed. Nevertheless, as it will be shown below, the system is already in inertial flow at the late stages and the contribution of the terms with the kinetic coefficients is negligible.

\section{Mechanisms of cluster formation}

As mentioned above, the linear stability analysis of the hydrodynamic equations predicts the instability of the homogeneous cooling state with respect to the density fluctuations, which leads to the formation of clusters. Let us discuss the basic mechanisms of the cluster formation, which we plan to check by numerical simulations. 
The linear stability analysis of the above hydrodynamic equations with the new kinetic coefficients may be performed in the same way as for the kinetic coefficients for the dilute limit, e.g. [5]. The obtained results are qualitatively the same, with a slight modification for the critical wavevectors, which demarcate stability regions for the transverse (shear) mode and for the heat mode [5]. More interesting however is the analysis of the non-linear cluster formation mechanisms, that reflect the background physics.

Goldhirsh and Zanetti suggested the mode-enslaving mechanism [15], which may be formulated as follows: Due to thermal fluctuations in the system, there always exists an initial shear mode with some wave-vector $\vec{k}$ (note that the flux velocity in a shear mode is perpendicular to $\vec{k}$ ). The amplitude of this random mode is generally very small, however its decay rate, proportional to $k^{2}$ is also very small for the long wave-length limit, $k \rightarrow 0$. For this reason the amplitude of the shear mode keeps practically constant for $k \rightarrow 0$, while the temperature of the gas permanently decreases. At some moment the thermal velocity of the gas, $v_{T} \equiv \sqrt{2 T / m}$, becomes comparable with the characteristic velocity of the shear mode $\vec{u}_{k \perp}(0)$. From this moment on, the heating due to the shear flux with viscosity $\eta$, which is estimated as $\eta\left(\nabla \vec{u}_{\perp}\right)^{2} \sim \eta k^{2}\left[\vec{u}_{k \perp}(0)\right]^{2}$ compensates the cooling of the gas which is estimated as $\sim \zeta T$. Thus the condition for the mode enslaving reads (note that $n T$ characterizes the energy density of the gas),

$$
\frac{\partial T}{\partial t}=-\zeta T+\frac{1}{n} \eta k^{2}\left[\vec{u}_{k \perp}(0)\right]^{2}=0
$$

or

$$
\zeta T=\frac{\eta}{n} k^{2}\left[\vec{u}_{k \perp}(0)\right]^{2} .
$$

Since $\zeta \propto\left(1-\varepsilon^{2}\right) n \sqrt{T}$ and $\eta \propto \sqrt{T}$ [see Eqs.(2.7,2.13,2.17)], we obtain for the enslaved temperature

$$
T \propto \frac{1}{n^{2}\left(1-\varepsilon^{2}\right)} .
$$

The balance between cooling and viscous heating is a local property, therefore the local temperature depends on the local density according to Eq. (3.3), and hence we obtain for the local pressure:

$$
p \propto n T \propto \frac{1}{n} .
$$

That is, the higher the density the smaller the pressure, which implies a negative compressibility and consequently an instability of the system with respect to the density fluctuations. Hence the mode-enslaving mechanism of the cluster formation predicts that temperature is inversely proportional to the square of density, while the pressure is inversely proportional to density. In other words, this mechanism of cluster formation entails specific correlations between density and pressure during the gas evolution. Let $\delta n(\vec{r})=n(\vec{r})-\langle n\rangle$ be the deviation of the density $n(\vec{r})$ form the average density $\langle n\rangle$ (which is constant, since the volume and number of particles is conserved). Similarly, let $\delta p(\vec{r})=p(\vec{r})-\langle p\rangle$ be the deviation of the pressure from its average value. Then the mode enslaving theory predicts the negative correlations between pressure and density fluctuations:

$$
\langle\delta p(\vec{r}) \delta n(\vec{r})\rangle \propto-\left\langle(\delta n(\vec{r}))^{2}\right\rangle\langle n\rangle^{-2} .
$$


These correlations between temperature and density and pressure and density, as well as the above relation (3.5) are checked in our numerical experiments.

\section{Numerical solution of the hydrodynamic equations}

The hydrodynamic Eqs. (2.1) have been solved numerically. As other authors have observed, we anticipate the occurrence of strong shocks across the system [1], and thus we discretize the granular Navier-Stokes equations using a WENO shock capturing scheme of fifth order for the advection terms, whereas the integration in time is a third order TVD Runge-Kutta [11]. This way monotonicity is preserved, as oscillations at the discontinuities are avoided. We use a $200 \times 200$ computational domain with periodic boundary conditions. The initial state is that of a Maxwellian gas at a given temperature and average density $\phi=0.2$ in packing fraction. These conditions have been taken from the mesoscopic average of a molecular dynamics simulation of a gas of elastic particles, in order to mimick the conditions in a particle numerical experiment. The constant coefficient of restitution is $\varepsilon=0.95$. The system size is imposed such that, for the parameters chosen, it neatly overcomes the critical size for cluster formation. As a matter of fact, if one analyzes the velocity field, the typical vortex instability is seen prior the development of clusters.

In Figures 1-6 the results of the numerical analysis are presented. Since evolution of a cooling granular gas slows down, it is convenient to measure time in the collision units per particle $\tau_{c}(t)$ :

$$
\tau_{c}^{-1}(t)=2 g_{2}(\sigma) \sigma n \sqrt{\frac{\pi T(t)}{m}} .
$$

Since the temperature and density of the gas are not necessarily homogeneous, we use the averaged value of $\tau_{c}(t)$. In Figure 1 we show the evolution of the density field of an initially homogeneous gas and in Figure 2 and Figure 3 the corresponding contour plots of temperature and pressure are shown along with the density field. As it follows from Figure 2, there are clearly visible negative correlations between the temperature of the gas and density: The temperature is smaller in denser regions, in agreement with the mode-enslaving mechanism. At the same time, as it may be seen from Figure 3, the pressure is always higher in dense regions, than in dilute regions. This is in a sharp contrast with the predictions of the mode enslaving theory, which implies $p \propto 1 / n$.

In Figure 4 (left) we plot the (normalized) correlation function for pressure and density fluctuations, $\langle\delta p(\vec{r}) \delta n(\vec{r})\rangle$ as the function of time, obtained in the numerical simulations, along with the predictions of the mode-enslaving theory, Eq. (3.5). Again, we see that the correlations are positive, while the mode-enslaving mechanism implies negative correlations. In the figure we also show the time evolution of the (normalized) mean square density fluctuation $\left\langle(\delta n)^{2}\right\rangle$, and the deviation of the average pressure from the ideal gas law, $\langle p / n T\rangle-1$. The latter allows to judge, whether the resultant pressure increase with density (positive pressure-density correlations) is to be attributed to the non-ideal gas contribution to the pressure. As it follows from Figure 4 (left), at the onset of cluster formation, a rapid growth of the density fluctuations and pressure deviation from the ideal gas law is observed. In Figure 4 (right) the same dependencies, but for a much more 

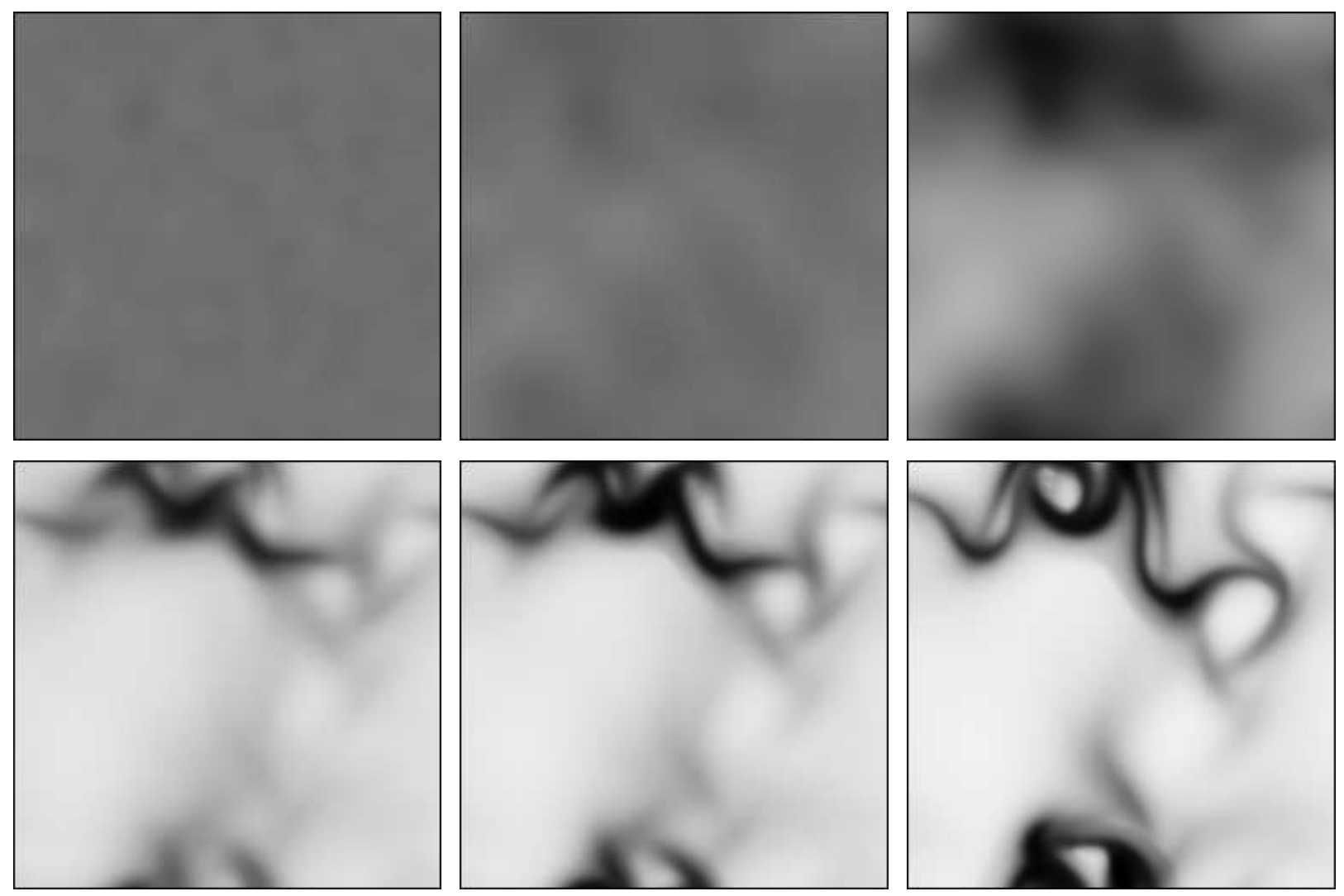

Figure 1: Density field obtained from the numerical solution of the hydrodynamic equations (2.1) for the gas with constant coefficient of restitution $\varepsilon=0.95$ and the average packing fraction $\phi=0.2$. The snapshots are taken for the time (in averaged number of collisions per particle) $\tau=$ 50, 200, 300, 400, 800 and 2200 (left to right, top to bottom). The formation and sharpening of clusters is clearly visible.

dilute gas with $\phi=0.02$ are shown. In the latter simulations we use, instead of Eq. (2.4), the ideal gas equation of state, $p=n T$. In Figure 4 (right) one observes the same behavior for the densitydensity and pressure-density correlations, as in the previous case of moderate gas density, $\phi=0.2$. This evidences that the failure of the mode-coupling theory to describe the density dependence of pressure is not related to the deviation of pressure from the ideal gas law.

Our simulation results demonstrate that pressure increases with density, and hence the positive feedback for the enhancement of density fluctuations is not confirmed. This implies that the mode-enslaving mechanism can not explain the cluster formation in force-free granular gases. To understand this phenomenon and to try to check other mechanisms of cluster formation, we plot the local values of the Mach number M. This quantity is defined as a ratio of the flow velocity $u$ and the local speed of sound $c$ : 

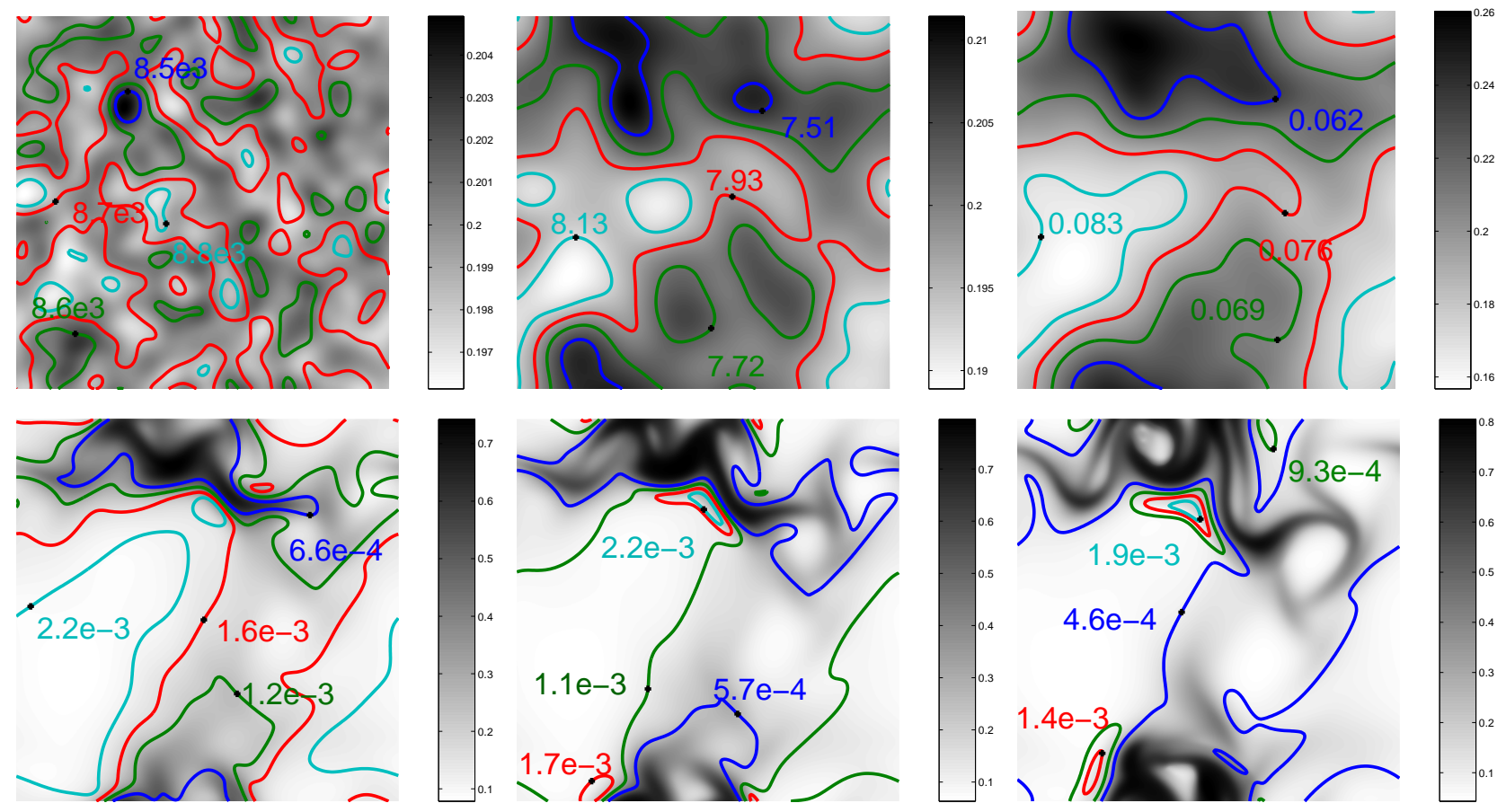

Figure 2: (Color online). Contour plots of temperature and density obtained for the same parameters and times as in Figure 1. In more dense (darker) regions the temperature is smaller. This result is in agreement with the notion that, inside the clusters, energy must decrease due to collisions.

$$
\mathbf{M} \equiv \frac{u}{c} \quad c=\sqrt{\frac{\partial p}{\partial \rho}+\frac{p}{\rho^{2}} \frac{\partial p}{\partial T}} .
$$

If $M>1$ the flow is supersonic, that is, the material moves faster than sound, the velocity of which corresponds to the maximal speed of propagation of any perturbation through the system. In Figure 5, evolution of the local values of the Mach number are shown as contour plots over the density. One can clearly see correlations between the location of clusters and the local Mach numbers: Near the cluster boundaries a rapid growth of $\mathbf{M}$ is observed, with a magnitude corresponding to the supersonic regime, $\mathbf{M}>1$. The regions of sharp (almost discontinuous) variation of density and the Mach number are specific to the front of a shock wave. Hence we conclude that clusters are formed mainly due to inertial flow. This mechanism of cluster formation has been observed in the one-dimensional [1] and quasi-one-dimensional [23] granular gases and has been also predicted for the dynamics of a cold gas of sticky particles [34].

Qualitatively, this may be understood as follows. Due to the dissipative collisions, the temperature of the gas quickly decreases; accordingly, the pressure and the compressibility of the gas (which defines the sound speed), quickly go down. At the same time the initial long wave-length fluctuations of the flow velocity, which always exist in the system, decay at much lower rate [25]. 

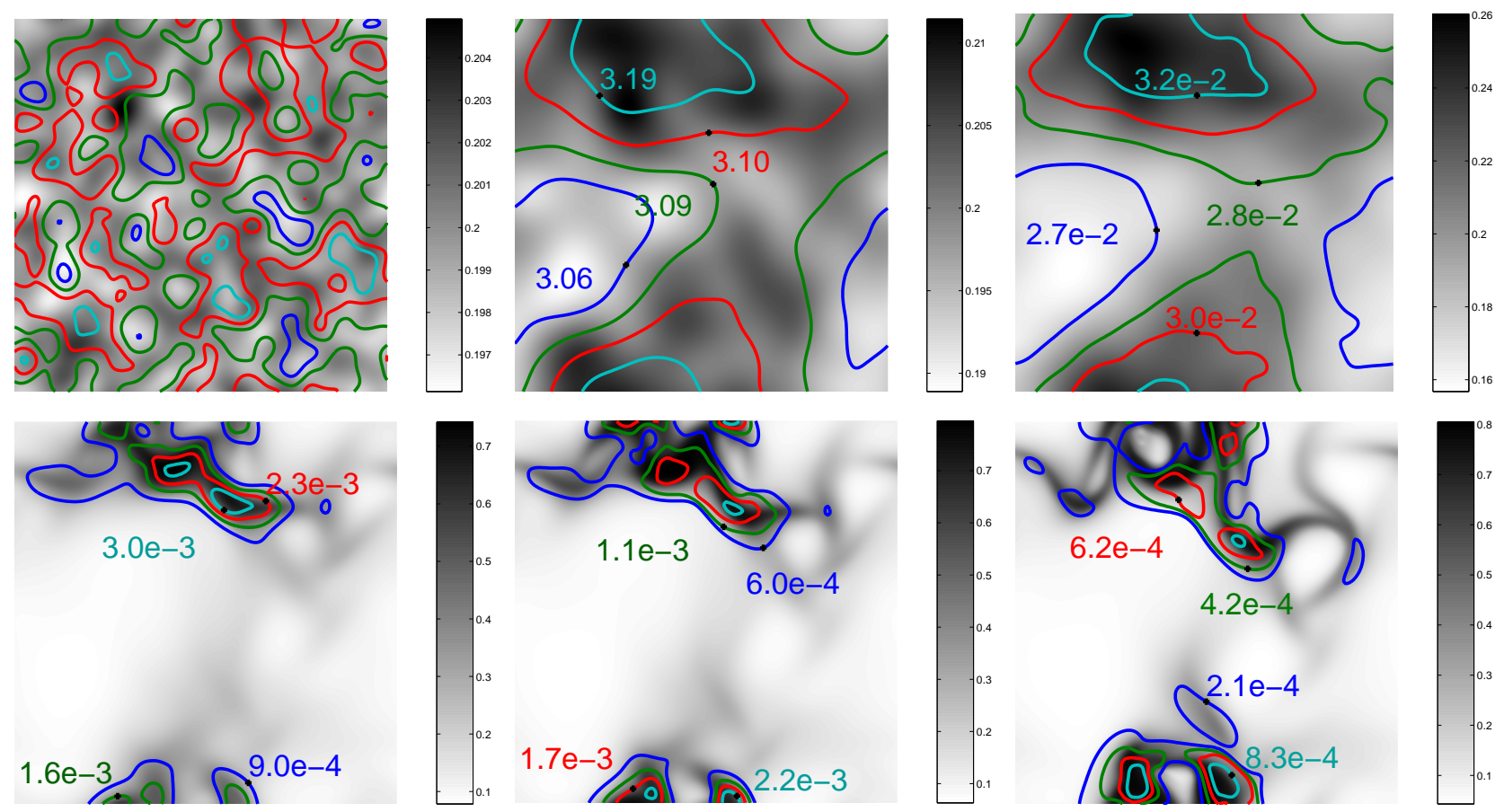

Figure 3: (Color online). The contour plots of pressure over the density field, obtained for the same parameters and times as in Figure 1. The pressure is higher in more dense (darker) regions. For the sake of clarity, the contour levels are suppressed on the top-left panel; the contour shapes, however, indicate that the variations of pressure are not correlated with density changes at the very early stages of cluster formation. Observe instead the direction of the pressure gradient immediately afterwards. When the density inhomogeneities across the system barely reach 5\%, the pressure is already higher in the more dense regions. In fact, no other trend is observed throughout the entire cluster formation phase. The slight pressure differences originated in the system after 200 collisions per particle (the middle figure in the top row of the panel) already show the general behavior of the pressure field: the pressure is always smaller in the dilute areas, and increases when approaching the packed phase. Thus, clusters incorporate particles and become denser against the surrounding pressure gradient. 

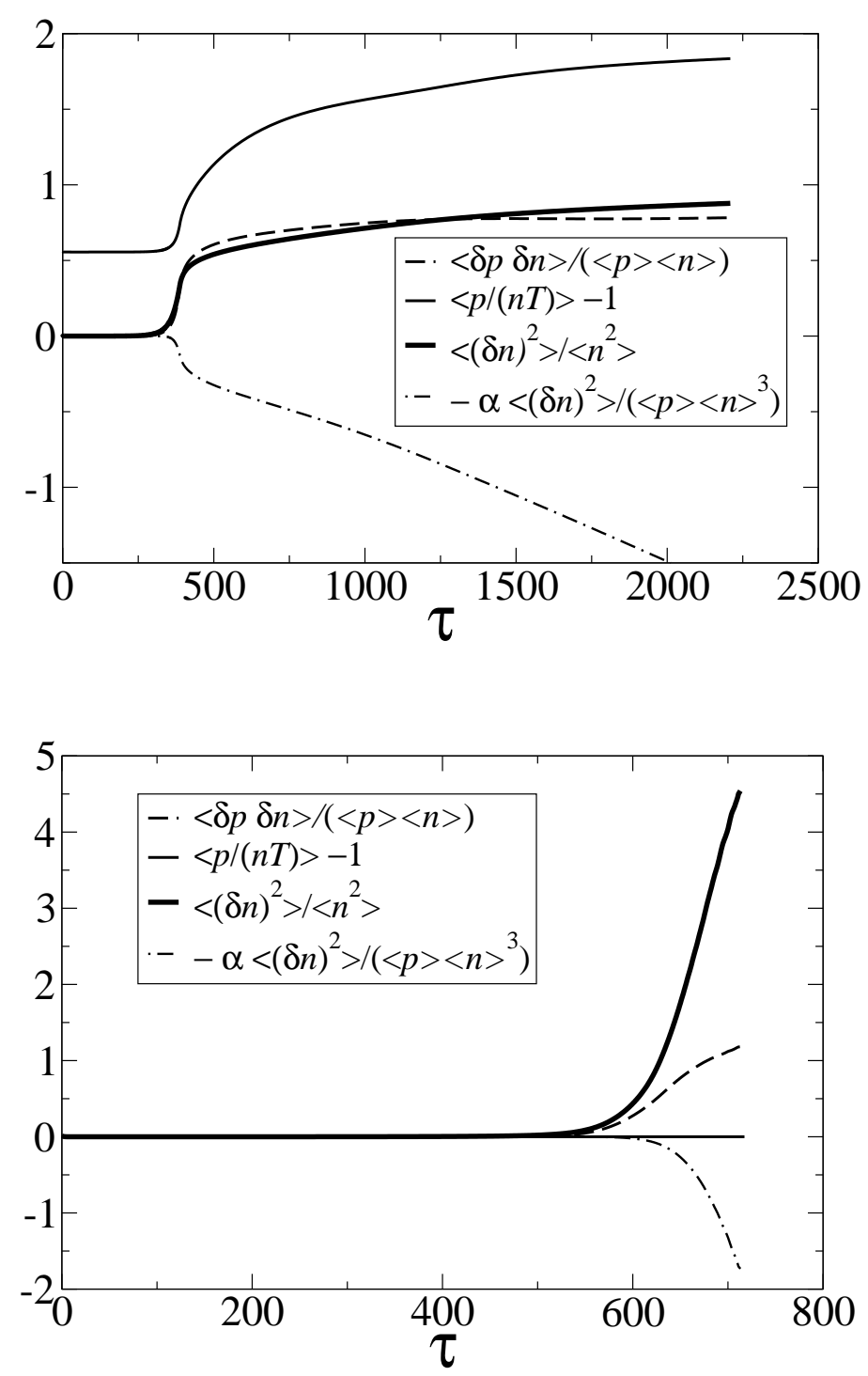

Figure 4: Time dependence of the normalized pressure-density correlations $\langle\delta p \delta n\rangle /(\langle p\rangle\langle n\rangle)$ and density-density correlations $\left\langle(\delta n)^{2}\right\rangle /\langle n\rangle^{2}$, together with the average deviation of pressure from the ideal-gas equation of state, $\langle p / n T\rangle-1$. The prediction of the mode-enslaving theory for the (normalized) pressure-density correlations, equal to $-\alpha\left\langle(\delta n)^{2}\right\rangle /\left(\langle p\rangle\langle n\rangle^{3}\right)$, is also shown. The coefficient $\alpha$ is chosen to depict all the dependencies on the same plot. Top panel: The granular gas of moderate density with the average packing fraction $\phi=0.2$ and $\alpha=5 \times 10^{-5}$. Bottom panel: The dilute granular gas with the average packing fraction $\phi=0.02$ and $\alpha=5 \times 10^{-7}$. For the dilute gas the ideal gas pressure, $p=n T$, for all densities is used, therefore the deviation $\langle p / n T\rangle-1$ is always zero in this case. Note that the pressure-density correlations are positive, whereas the mode-enslaving theory predicts negative sign for these correlations. Also note that the positive sign of the pressure-density correlations does not steam from the deviation of pressure from the ideal-gas law, which is illustrated in the right panel. 

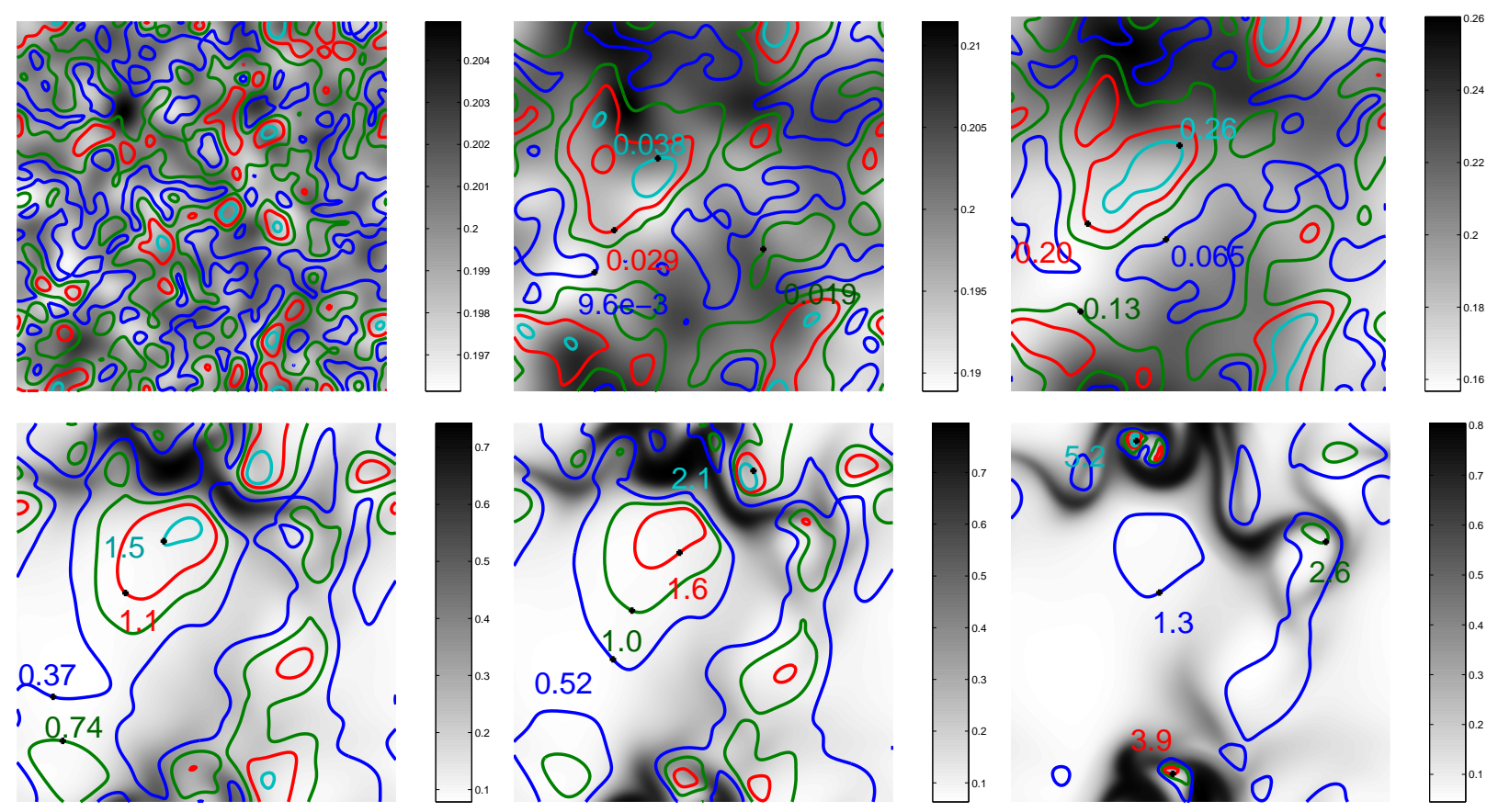

Figure 5: (Color online). The contour plots of the Mach number (Eq. 4.2) and the density, obtained for the same parameters and times as in Figure 1. A significant increase of the Mach number in the close vicinity of the cluster boundaries is clearly seen. The contour levels are suppressed on the top-left panel for the same reason as in Figure 3.

Indeed, they are conserved variables, so that their time derivative vanishes in the long wave-length limit $k \rightarrow 0$ [31]. As a result, shock waves are formed. This happens because in the supersonic regime the system can not respond to any perturbation, that is, its response is too slow on the time scale of the supersonic motion. Therefore any initial dispersion of the flow velocity due to fluctuations leads eventually to steep density gradients [34], i.e. to clustering. Hence the distribution of pressure is not of a primary importance in this regime. For the case of $\varepsilon=$ const, the system always cools down faster than the velocity fluctuations decay. Therefore the Mach number increases (see Figures 5, 6), exceeds 1 and then keeps supersonic, $\mathbf{M}>1$. Correspondingly the clusters, once formed, persist and their boundaries become sharper. The existing pressure gradients are too small and can not counteract the formation of clusters due to the shock waves. In Figure 6 the process is shown through the plots of the Mach number and the density. At the beginning there is a diffusive phase with no clusters. Then, the instability develops. It happens between 300 and 400 collisions per particle, as it may be seen from the maximal density and square average density deviation curves. Interestingly, this time precisely coincides with the instant, where $M=1$ is achieved (somewhere) in the system. Another regime starts when density reaches its maximal close-packing value $\phi_{\mathrm{c}}$ at some other spot (these spots are small regions surrounded by less dense material). At this moment, the average Mach number is about 0.5 with ample regions in the system traveling at supersonic speeds. From this point on, the Mach number as well as the square average 
density deviation grow slower, until the blowup of the Mach number at about $\tau=2200$ collisions occurs.

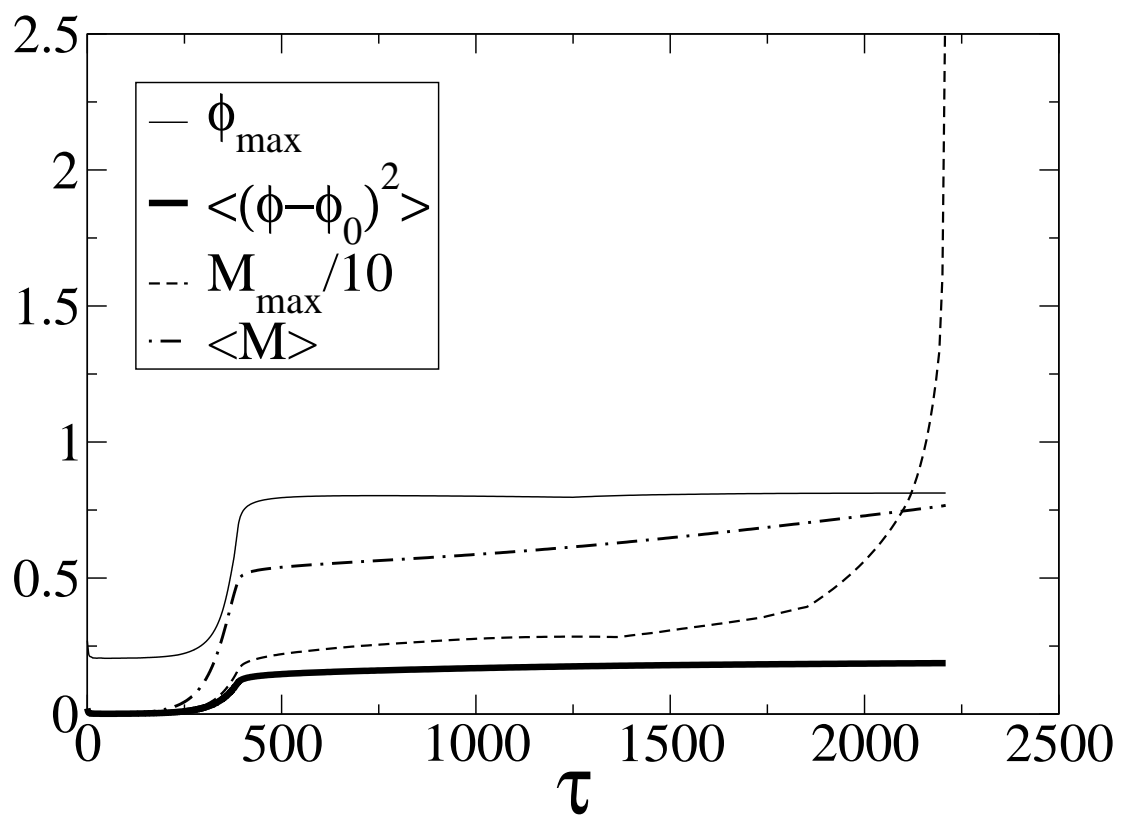

Figure 6: Evolution of the current maximal packing fraction $\phi_{\max }$ (not to be mixed up with the maximal close-packing value $\left.\phi_{\mathrm{c}}\right)$, its square average deviation $\left\langle\left(\phi-\phi_{0}\right)^{2}\right\rangle$, the maximal Mach number $\mathbf{M}_{\max }$ and the average Mach number $\langle\mathbf{M}\rangle$. Time is given in average number of collisions. Full lines: thin $-\phi_{\max }$, thick $-\left\langle\left(\phi-\phi_{0}\right)^{2}\right\rangle$; dashed lines $-\mathbf{M}_{\max }$ and dashed-dot lines $-\langle\mathbf{M}\rangle$. The parameters of the systems are the same as in Figure 1.

\section{Conclusion}

Kinetics of cluster formation in a force-free granular gas is investigated. We solve numerically the granular hydrodynamics equations and compare the simulation results obtained with the predictions of the mode-enslaving theory of cluster formation. We reveal that the correlations between pressure and density, predicted by the mode-enslaving theory, are not observed in granular gases: On the contrary, the pressure is always higher in dense regions than in dilute ones. Therefore, the positive feedback for the enhancement of the density fluctuation is not formed, that is, the modeenslaving mechanism can not explain the process of cluster formation. This conclusion has been confirmed by our simulations for the other gas density, which corresponds to the very dilute limit.

To understand the mechanism of cluster formation we analyze the time-dependence of the local Mach number, which serves as an indicator for the formation of shock waves. We observe direct correlations between the appearance of shock-waves and formation of clusters. Hence we conclude that cluster formation is driven by the shock-waves which are initiated by the initial fluctuations 
of the flux velocity in a homogeneous system. Such mechanism corresponds to the "scenario number 3" of cluster-formation, discussed in [29], mainly for quasi-one dimensional systems. Our conclusion is also consistent with the conjecture made by Ben-Naim et al [1] for one-dimensional granular gases.

Although we have shown that the cluster formation in our systems - moderately dense and very dilute force-free granular gases, may be attributed to shock-wave formation and propagation, we can not exclude that the mode-enslaving mechanism may be relevant for other systems, e.g. for driven systems, where a negative compressibility has been observed [19]. This possibility is to be checked in future studies.

\section{Acknowledgements}

The authors are thankful to V. Garzó for valuable discussions and for providing his preprint [13] and to the KIT programm of the Isaac Newton Institute of Mathematical Science for financial support and a creative scientific atmosphere. C.S acknowledges support from the Spanish Ministry of Science and Education, under grant of the National Program for Human Resources I+D+I 20082011. L.A is supported by I-MATH Research Grant FUT-C4-0176.

\section{References}

[1] E. Ben-Naim, S. Y. Chen, G. D. Doolen, S. Redner. Shock-like dynamics of inelastic gases. Phys. Rev. Lett., 83 (1999), 4069-4072.

[2] J. J. Brey, D. Cubero. Hydrodynamic transport coefficients of granular gases. In Pöschel and Luding [28], 59-78.

[3] J. J. Brey, J. W. Dufty, C. S. Kim, A. Santos. Hydrodynamics for granular flow at low density. Phys. Rev. E, 58 (1998), 4638-4653.

[4] J. J. Brey, M. J. Ruiz-Montero, D. Cubero. Origin of density clustering in a freely evolving granular gas. Phys. Rev. E, 60 (1999), 3150-3157.

[5] N. V. Brilliantov, T. Poeschel. Kinetic Theory of Granular Gases. University Press, Oxford, 2004.

[6] N. V. Brilliantov, T. Pöschel. Hydrodynamics of granular gases of viscoelastic particles. Phil. Trans. R. Soc. Lond. A, 360 (2001), 415-428.

[7] N. V. Brilliantov, T. Pöschel. Hydrodynamics and transport coefficients for granular gases. Phys. Rev. E, 67 (2003), 061304.

[8] N. V. Brilliantov, C. Saluena, T. Schwager, T. Pöschel. Transient structures in a granular gas. Phys. Rev. Lett., 93 (2004), 134301. 
[9] N. V. Brilliantov, F. Spahn, J.-M. Hertzsch, T. Pöschel. Model for collisions in granular gases. Phys. Rev. E, 53 (1996), 5382-5393.

[10] R. Brito, M. H. Ernst. Extension of Haff's cooling law in granular flows. Europhys. Lett., 43 (1998), 497-504.

[11] J. A. Carrillo, T Pöschel, C. Salueña. Granular hydrodynamics and pattern formation in vertically oscillated granular disk layers. J. Fluid Mech., 597 (2008), 119-144.

[12] E. Efrati, E. Livne, B. Meerson. Hydrodynamic singularities and clustering instability in a freely cooling inelastic gas. Phys. Rev. Lett., 94 (2005), 088001.

[13] V. Garzo. Enskog constitutive equations for hard disks. preprint (2008).

[14] V. Garzo, J. W. Dufty. Dense fluid transport for inelastic hard spheres. Phys. Rev. E, 59 (1999), 5895-5911.

[15] I. Goldhirsch, G. Zanetti. Clustering instability in dissipative gases. Phys. Rev. Lett., 70 (1993), 1619-1622.

[16] A. Goldshtein, M. Shapiro. Mechanics of collisional motion of granular materials. Part 1: General hydrodynamic equations. J. Fluid Mech., 282 (1995),75-114.

[17] S. A. Hill, G. F. Mazenko. Granular clustering in a hydrodynamic simulation. Phys. Rev. E, 67 (2003), 061302.

[18] J. T. Jenkins, M. W. Richman. Grad's 13-moment system for a dense gas of inelastic spheres. Archives for Particle Mechanics and Analysis, 87 (1985), 355-377.

[19] E. Khain, B. Meerson. Symmetry-breaking instability in a prototypical driven granular gas. Phys. Rev. E, 66 (2002), 021306.

[20] G. Kuwabara, K. Kono. Restitution coefficient in a collision between two spheres. Jpn. J. Appl. Phys., 26 (1987), 1230-1233.

[21] C. K. K. Lun, S. B. Savage, D. J. Jeffrey, N. Chepurniy. Kinetic theories for granular flow: Inelastic particles in Couette flow and slightly inelastic particles in a general flowfield. J. Fluid Mech., 140 (1984), 223-256 .

[22] J. F. Lutsko. Transport properties of dense dissipative hard-sphere fluids for arbitrary energy loss models. Phys. Rev. E, 72 (2005), 021306.

[23] B. Meerson, A. Puglisi. Towards a continuum theory of clustering in a freely cooling inelastic gas. Europhys. Lett., 70 (2005), 478-484.

[24] W. A. M. Morgado, I. Oppenheim. Energy dissipation for quasielastic granular particle collisions. Phys. Rev. E, 55 (1997), 1940-1945. 
[25] X. Nie, E. Ben-Naim, S. Y. Chen. Dynamics of freely cooling granular gases. Phys. Rev. Lett., 89 (2002), 204301.

[26] T. Pöschel, N. V. Brilliantov, editors. Granular Gas Dynamics, Lecture Notes in Physics Vol. 624. Springer, Berlin, 2003.

[27] T. Pöschel, N. V. Brilliantov, T. Schwager. Long-time behavior of granular gases with impactvelocity dependent coefficient of restitution. Physica A, 325 (2003), 274-283.

[28] T. Pöschel, S. Luding, editors. Granular Gases, Lecture Notes in Physics Vol. 564. Springer, Berlin, 2001.

[29] A. Puglisi, M. Assaf, I. Fouxon, B. Meerson. Attempted density blowup in a freely cooling dilute granular gas: Hydrodynamics versus molecular dynamics. Phys. Rev. E, 77 (2008), 021305.

[30] R. Ramírez, T. Pöschel, N. V. Brilliantov, T. Schwager. Coefficient of restitution for colliding viscoelastic spheres. Phys. Rev. E, 60 (1999), 4465-4472.

[31] P. Resibois, M. de Leener. Classical Kinetic Theory of Fluids. Wiley \& Sons, New York, 1977.

[32] T. Schwager, T. Pöschel. Coefficient of restitution of viscous particles and cooling rate of granular gases. Phys. Rev. E, 57 (1998), 650-654.

[33] N. Sela, I. Goldhirsch. Hydrodynamic equations for rapid flows of smooth inelastic spheres, to Burnett order. J. Fluid Mech., 361 (1998), 41-74.

[34] S. F. Shandarin, Ya. B. Zeldovich. The large-scale structure of the universe: Turbulence, intermittency, structures in a self-gravitating medium. Rev. Mod. Phys., 61 (1989), 185-222.

[35] F. Spahn, U. Schwarz, J. Kurths. Clustering of granular assemblies with temperature dependent restitution and under keplerian differential rotation. Phys. Rev. Lett., 78 (1997), 1596-1599.

[36] S. Torquato. Nearest-neighbor statistics for packings of hard spheres and disks. Phys. Rev. E, 51 (1995), 3170-3555. 